\title{
Detection of superhumps in the Z Camelopardalis-type dwarf nova AT Cnc at standstill`
}

\author{
V. P. Kozhevnikov $\star \star$ \\ Astronomical Observatory, Ural State University, Lenin Av. 51, Ekaterinburg 620083, Russia \\ Received 30 October 2003 / Accepted 26 January 2004

\begin{abstract}
We present results of our photometry of the Z Camelopardalis-type dwarf nova AT Cnc. The observational data were obtained during 13 nights in February and March 2003 when AT Cnc was in its long standstill. Two sets of our data reveal brightness variations with quasi-periods of $(4.65 \pm 0.02)$ and of $(4.74 \pm 0.02) \mathrm{h}$. The semiamplitude of these variations was observed in the range 5-9 mmag, showing changes from night to night. This signal varies in period and in phase on a time-scale of weeks, as is typical of superhumps. A comparison with the orbital period obtained by Nogami et al. (1999) from the radial velocity measurements of AT Cnc, which equals $(4.826 \pm 0.014) \mathrm{h}$, shows that this signal can be a negative superhump since the average period of brightness variations is approximately $3 \%$ shorter than the orbital period. These results make AT Cnc a permanent superhump system with a rather large orbital period and a large mass ratio. This is also the first detection of superhumps in a Z Camelopardalis-type system. In addition, we have found signs of an unstable signal with a period of about $2.3 \mathrm{~h}$, which may represent the second harmonic of the 4.7-h signal or the second orbital sideband of the wobble frequency of the tilted precessing accretion disc. The average power spectrum of AT Cnc reveals a broad hump at frequencies in the range $0.4-0.7 \mathrm{mHz}$, which is evidence of quasiperiodic oscillations. The hump crest corresponds to a period of 1700-1800 s, which is remarkably close to $10 \%$ of the orbital period. This may indicate that this system contains a magnetic white dwarf creating the tilted accretion disc.
\end{abstract}

Key words. stars: individual: AT Cnc - stars: novae, cataclysmic variables - stars: oscillations

\section{Introduction}

Cataclysmic variables (CVs) are interacting binaries that consist of a white dwarf primary accreting matter from a low mass secondary filling its Roche lobe. The path that the transferred matter takes depends strongly on the magnetic field of the white dwarf. A bright accretion disc forms in non-magnetic systems, while matter swirling along field lines releases energy in their magnetic counterparts. Usually CVs show a variety of periodic, quasiperiodic and aperiodic brightness variations. Among the CVs, perhaps the most interesting class is the intermediate polars (or DQ Herculis stars) - systems displaying a photometric variation at a period shorter than, and usually much shorter than, the orbital period. This period represents the rotation period of a magnetic white dwarf and therefore is extremely stable. Properties of intermediate polars are reviewed by Patterson (1994). Many CVs of short orbital period have lightcurves with prominent humps, which reveal periods slightly longer than the orbital period. These are called "superhumps" since they are characteristic of the SU Ursae Maioris-type dwarf novae in superoutburst. Some CVs show superhumps in their optical lightcurves during the normal brightness state. In most cases

\footnotetext{
* Research supported in part by the scientific program "Russian Universities" under grant No. UR.02.01.024.

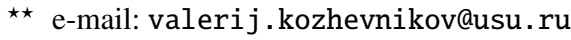

such CVs are bright nova-like variables and nova remnants (Osaki 1996; Retter \& Naylor 2000). These superhumps are called permanent superhumps. Permanent superhumps can either be a few per cent longer than the orbital periods and they are called "positive superhumps", or they can be a few per cent shorter - "negative superhumps". Typical full amplitudes of permanent superhumps are about 5\%-15\%, but they are highly variable and sometimes even disappear from the lightcurve. The periods of the superhumps are unstable and usually show appreciable wobbling. The positive superhump is explained as the beat between the binary motion and the precession of an eccentric accretion disc in the apsidal plane. The negative superhump is explained as the beat between the orbital period and the nodal precession of the disc tilted to the orbital plane. A review of permanent superhumps is given by Patterson et al. (1993). Some recent data about permanent superhumps are given by Patterson et al. (2002).

AT Cnc is a Z Camelopardalis-type dwarf nova that shows normal outbursts and standstills typical of this subtype of CVs. The long-term lightcurve of AT Cnc between 1995 and 2003 was obtained from the visual observations of VSNET network observers (http://vsnet.kusastro.kyoto-u.ac.jp/ vsnet/LCs/index/CNCAT.html). This lightcurve is presented in Fig. 1. Looking at this lightcurve, we can learn that the visual magnitude of AT Cnc changes in the range 


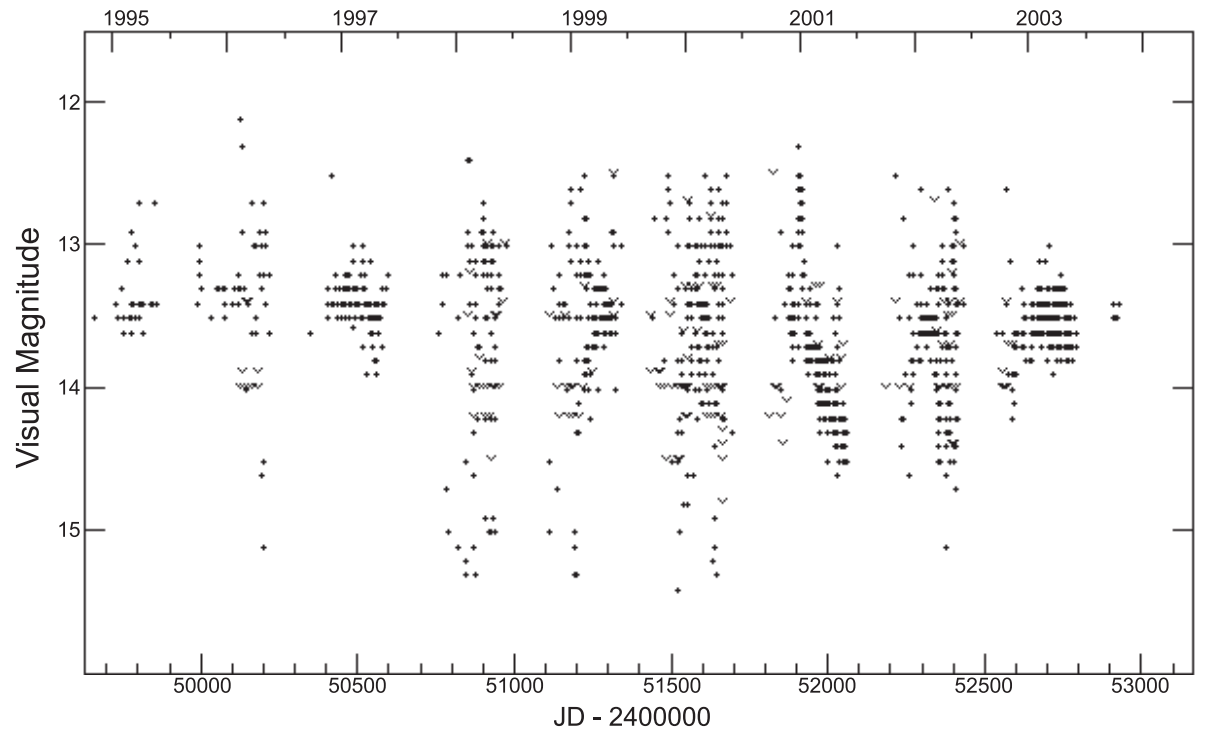

Fig. 1. Long-term lightcurve of AT Cnc. This lightcurve is obtained from the visual observations of VSNET network observers. Crosses and "v" symbols represent positive observations and upper limits, respectively.
12.1-15.4. The outbursts and standstills of this dwarf nova are usually short (a few weeks), but sometimes AT Cnc reveales very long standstills having durations of about half a year. Such long standstills were observed in 1996-1997 and in 2002-2003. During these standstills the visual magnitude of AT Cnc was observed in the range 13-14. Extensive photographic observations of AT Cnc were obtained by Götz (1983, 1985, 1986, 1988a,b, 1990, 1991). Götz (1986) detected photometric variations with a period of $0.2386913 \mathrm{~d}$ and attributed these variations to the orbital motion of AT Cnc. Götz (1988a,b, 1990, 1991) confirmed this oscillation but suggested that its shape had varied. Spectroscopic observations that could reveal the orbital motion of AT Cnc were conducted by Nogami et al. (1999). They found the orbital period of the system equal to $(0.2011 \pm 0.0006) \mathrm{d}$. In addition, their photometry revealed an oscillation with a semiamplitude of $0.01 \mathrm{mag}$ and with period candidates of $0.132,0.124,0.142$ and $0.249 \mathrm{~d}$. These period candidates turned out to be too short or too long in comparison with the orbital period. Hence, the observed oscillation could not be the possible superhumps in the lightcurve of AT Cnc. Therefore, Nogami et al. suggested that the oscillation may be caused by asynchronous rotation of a magnetic white dwarf, i.e. AT Cnc may be an intermediate polar. To confirm the oscillation found by Nogami et al., to define more reliably the oscillation period and to establish its stability, we performed photometric observations of AT Cnc. In this paper, we present results from our observations spanning a total duration of $97 \mathrm{~h}$.

\section{Observations}

AT Cnc was observed in February and March 2003 during 13 nights, using a three-channel (two stars + sky) photometer attached to the $70-\mathrm{cm}$ telescope at the Kourovka observatory of the Ural State University. A journal of the observations is given in Table 1. The program and nearby comparison stars were observed through $16^{\prime \prime}$ diaphragms in the first and second channels, and the sky background was observed in the third channel. The comparison star is located at the direction SSW
Table 1. Journal of the observations.

\begin{tabular}{crrr}
\hline \hline $\begin{array}{c}\text { Date } \\
\text { (UT) }\end{array}$ & $\begin{array}{c}\text { HJD start } \\
(-2452000)\end{array}$ & \multicolumn{1}{c}{$\begin{array}{c}\text { Length } \\
(\mathrm{h})\end{array}$} \\
\hline 2003 Feb. 3 & 674.15046 & 10.22 \\
2003 Feb. 8 & 679.11439 & 9.89 \\
2003 Feb. 9 & 680.11676 & 9.94 \\
2003 Feb. 10 & 681.13162 & 10.22 \\
2003 Feb. 11 & 682.12815 & 9.33 \\
2003 Feb. 20 & 691.12969 & 5.50 \\
2003 Feb. 24 & 695.18923 & 2.44 \\
2003 Feb. 26 & 697.18928 & 7.83 \\
2003 Feb. 27 & 698.13643 & 3.67 \\
2003 Mar. 3 & 702.21418 & 7.28 \\
2003 Mar. 8 & 707.14514 & 6.17 \\
2003 Mar. 9 & 708.16311 & 7.33 \\
2003 Mar. 10 & 709.15111 & 6.78 \\
\hline
\end{tabular}

and at a separation of 10'2 from AT Cnc. Data were registered at 0.5 -s exposure times (the dead times are only $20 \mu \mathrm{s}$ ) in white light (approximately 3000-8000 ̊), employing a PC based data acquisition system. Next, the data were collected at 8-s sampling times. The design of the photometer is described by Kozhevnikov \& Zakharova (2000). We recently incorporated a CCD guiding system into the photometer. This guiding system enables precise centering of the two stars in the diaphragms to be maintained automatically during an observational night and facilitates the acquisition of long lightcurves.

The continuous measurements of the sky background were subtracted from the program and comparison star data, taking into account the differences in light sensitivity between the channels. Then we took differences of magnitudes of the program and comparison stars. Because the angular separation between the program star and comparison star is only $10^{\prime}$, the differential magnitudes are corrected for first order atmospheric extinction and light absorption by thin clouds that appeared sometimes during the observations. 


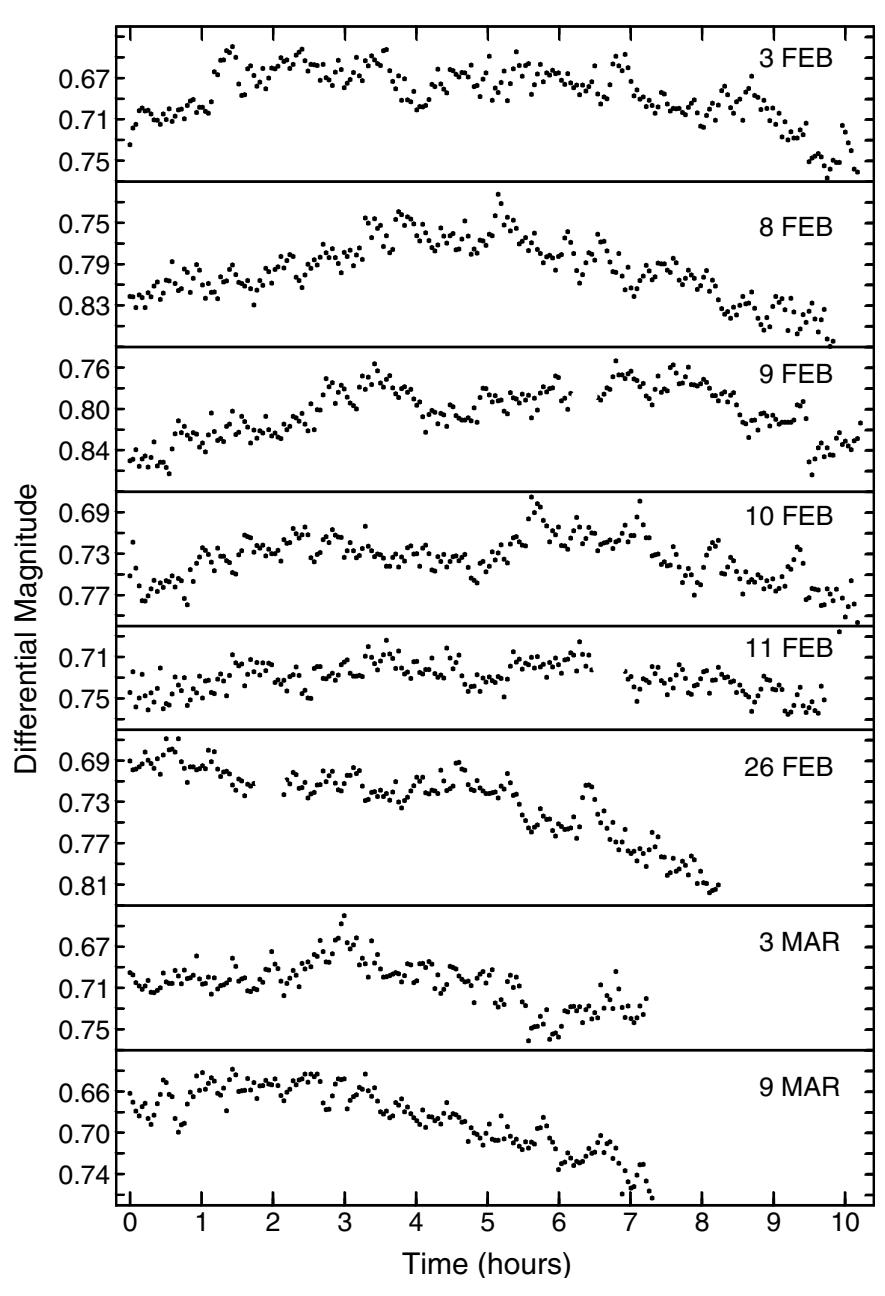

Fig. 2. Differential lightcurves of AT Cnc. The magnitudes are averaged over 152-s time intervals. The rms noise of the lightcurves is 4-6 mmag.

The mean counts are 14300, 21700, 5800 and 5300 for AT Cnc, the comparison star and the sky background in the first and second channels, accordingly. These counts result in the photon noise (rms) of the differential lightcurves, which equals $18 \mathrm{mmag}$. The actual rms noise of the differential lightcurves includes also the noises due to atmospheric scintillations and motion of the star images in the diaphragms. We estimate the scintillation noise roughly equal to $5 \mathrm{mmag}$. Unfortunately, the noise caused by motion of the star images was variable. Due to low altitudes above the horizon, the scatter of points in some parts of the long lightcurves was found slightly larger than in the best conditions. We estimate this noise approximately equal to the photon noise in these cases and is negligible at high altitudes. The total rms noise of the lightcurves is 19-26 mmag. Figure 2 presents the longest differential lightcurves of AT Cnc with magnitudes averaged over 152-s time intervals. The rms noise of these lightcurves is smaller by a factor of $\sqrt{19}$ and equal to 4-6 mmag. Besides the white noise components every photometric system usually shows the $1 /$ f noise component which decreases the precision at frequencies approximately below $1 \mathrm{mHz}$ (e.g., Young et al. 1991). This noise component in our photometer is approximately equal to $1 \mathrm{mmag}$. A detailed description of the noise analysis carried out for the photometer is given by Kozhevnikov \& Zakharova (2000).

\section{Analysis and results}

As seen in Fig. 2, the lightcurves of AT Cnc are fairly typical of $\mathrm{CVs}$ in showing rapid flickering. In addition, some of them show prominent maxima and minima, which may indicate periodic oscillation on a time-scale of hours. Lowfrequency trends, which are apparently caused by differential extinction, are also visible in the lightcurves. These trends were removed by subtraction of a second order polynomial fit to each lightcurve. We then calculated the amplitude spectrum for each long lightcurve, using a fast Fourier transform (FFT) algorithm. Our longest lightcurve consists of 242 points (152-s time resolution). Since the FFT algorithm can operate only if lightcurves have a length of a power of 2 , the lightcurves were padded with zeroes up to 256 points. The corresponding amplitude spectra consist of 128 frequency components with frequency bins of $0.025699 \mathrm{mHz}$. These amplitude spectra are shown in Fig. 3. Most of them display prominent peaks at the second and third frequency components which correspond to oscillation frequencies of $(0.051 \pm 0.013)$ and $(0.077 \pm 0.013) \mathrm{mHz}$. We consider the error equal to half the frequency bin. If these peaks indicate an oscillation, its frequency is between these two frequency components, i.e. this frequency is $(0.064 \pm 0.026) \mathrm{mHz}$ and corresponds to a period in the range 3.1-7.3 h. As seen in the amplitude spectra, the semiamplitude of the oscillation is in the range 5-9 mmag and variable from night to night. However, since the length of the individual lightcurves is only 1.5-2.0 oscillation cycles, the applied de-trending slightly decreases the amplitude. The real semiamplitude of this oscillation is approximately $20 \%$ larger. Some other amplitude spectra (Fig. 3) reveal the peaks at the fifth and sixth frequency components. These peaks may indicate an oscillation at a frequency of $(0.141 \pm 0.026) \mathrm{mHz}$. This frequency corresponds to a period in the range $1.7-2.4 \mathrm{~h}$.

To evaluate the statistical significance of detection of these oscillations, we calculated an average power spectrum. The amplitude spectra were divided by $\sqrt{2}$ and squared. We then took an average of these squared amplitude spectra. The average power spectrum is given in Fig. 4. Two large peaks at frequencies of 0.064 and $0.141 \mathrm{mHz}$ are easily visible in this power spectrum. Usually the probability that a peak in a power spectrum is a chance noise fluctuation may be estimated by the peak height relative to the surrounding noise level. However, it seems useless to estimate the probability that each of these two peaks is a chance noise fluctuation since these wide peaks may simply indicate the continuous rise of power at lower frequencies. The flickering may cause such a rise. In this case, it seems relevant to estimate the probability that the falling of the fourth frequency component separating these two peaks is a chance noise fluctuation, taking the average height of these two peaks as the noise level. Taking into account that each frequency component in an average power spectrum is distributed as $\chi^{2}(v) / v$, where $v$ is the number of degrees of freedom (e.g., Bendat \& Piersol 1986), we can find this probability. In our case the number of degrees of freedom, $v$, equals 16 because of 


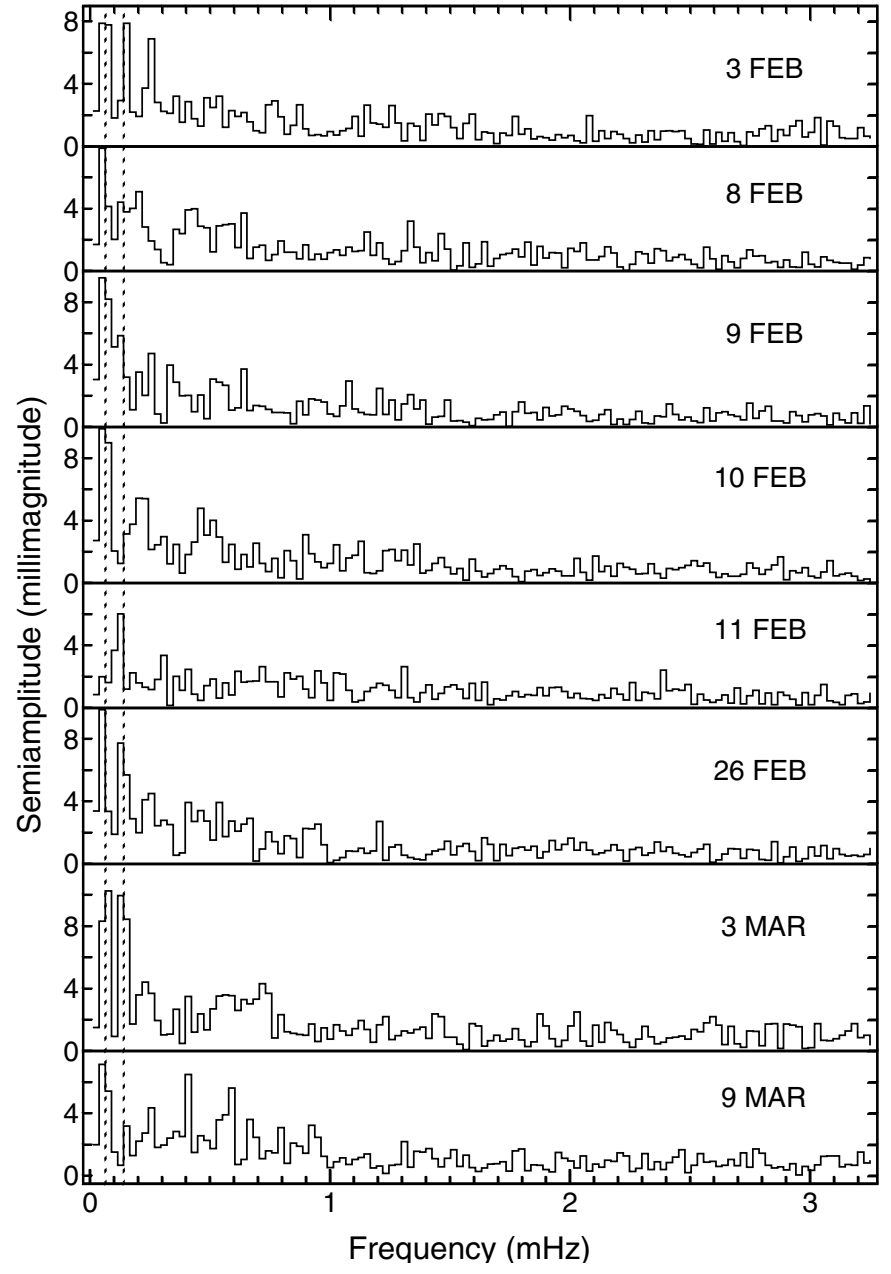

Fig. 3. Amplitude spectra of AT Cnc. Most of them display the prominent peaks at the second and third frequency components which indicate an oscillation at a frequency of $(0.064 \pm 0.026) \mathrm{mHz}$. Some amplitude spectra reveal peaks at the fifth and sixth frequency components. These peaks may indicate an oscillation at a frequency of $(0.141 \pm 0.026) \mathrm{mHz}$. Dotted lines mark the corresponding frequencies.

averaging of eight power spectra. For the decrease of the fourth frequency component, this probability is less than $0.05 \%$. Thus, the oscillations at frequencies of 0.064 and $0.141 \mathrm{mHz}$ seem real.

The two oscillations revealed in the average power spectrum (Fig. 4) have only a few oscillation cycles in each individual lightcurve. Therefore, the evaluation of the oscillation periods and of the phase coherence with a tolerable accuracy seems impossible, using the individual power spectra or the average power spectrum. To evaluate more accurately the periods of the observed oscillations and to define their stability, we calculated power spectra using the lightcurves together and the lightcurves divided into two groups, excluding the two short lightcurves obtained in 24 February and in 27 February. These groups included the data obtained in the time intervals between 3 February and 20 February and between 26 February and $10 \mathrm{March}$. The total durations of the observations for these two groups are $55 \mathrm{~h}$ and $36 \mathrm{~h}$, accordingly. Then we constructed three time series consisting of these lightcurves. As before, the low frequency trends were removed by subtraction of

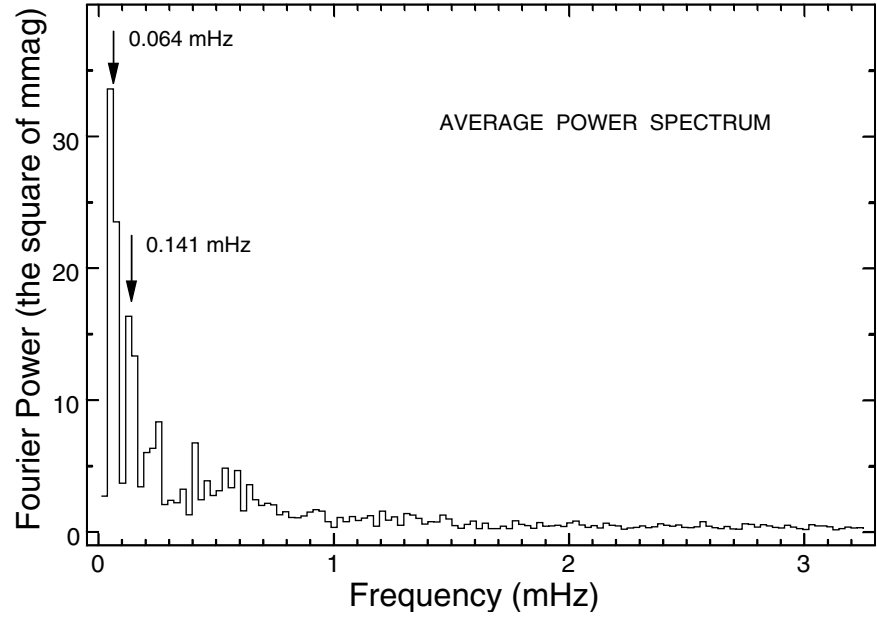

Fig. 4. Average power spectrum of AT Cnc. Two large peaks at frequencies of $(0.064 \pm 0.026)$ and $(0.141 \pm 0.026) \mathrm{mHz}$ are visible in this power spectrum.

a second order polynomial fit to each individual lightcurve. The magnitudes were averaged over 200-s time intervals. All the gaps caused by the absence of observational data were padded with zeroes. In addition, these three time series consisting of 15243,7434 and 5289 points were padded with zeroes up to 16384 points for all the data sets together and up to 8192 points for the two groups of the data. The power spectra were calculated using the FFT algorithm. Each of these power spectra consists of 8192 frequency components with frequency bins of $0.000305 \mathrm{mHz}$ for all the data sets together and of 4096 frequency components with frequency bins of $0.000610 \mathrm{mHz}$ for the data sets combined into two groups. Low-frequency regions of these power spectra are presented in Fig. 5. In the range $0.03-0.09 \mathrm{mHz}$ these power spectra reveal distinct pictures of principal peaks and one-day aliases proving the existence of the oscillation.

While within each of the two groups of the data the oscillation seems nearly coherent, the spectrum in the lower frame shows that this oscillation has a low degree of coherence since the principal peak and the one-day aliases have multicomponent structures, which do not conform to the window function when the oscillation is considered within all the observations. Since the frequency composition of a strictly periodic oscillation can be characterized by a delta-function, any principal peak corresponding to such an oscillation must have the width coinciding with the frequency resolution, which improves with the extension of the observations. If we calculate a power spectrum using the FFT algorithm, the frequency resolution simply coincides with the frequency bin when the length of the time series is close to a power of 2 . That is why we expect the single-component structures of the principal peaks and the one-day aliases in the Fourier power spectra for the strictly periodic oscillations. For example, just such a behaviour of the power spectra was observed by us in the suspected cataclysmic variable NSV 2872 which revealed the coherent oscillation with a period of 87.65 min (Kozhevnikov 2003). In addition, the oscillation frequencies of AT $\mathrm{Cnc}$ are found equal to $(0.0598 \pm 0.0003) \mathrm{mHz}$ within the time interval between 


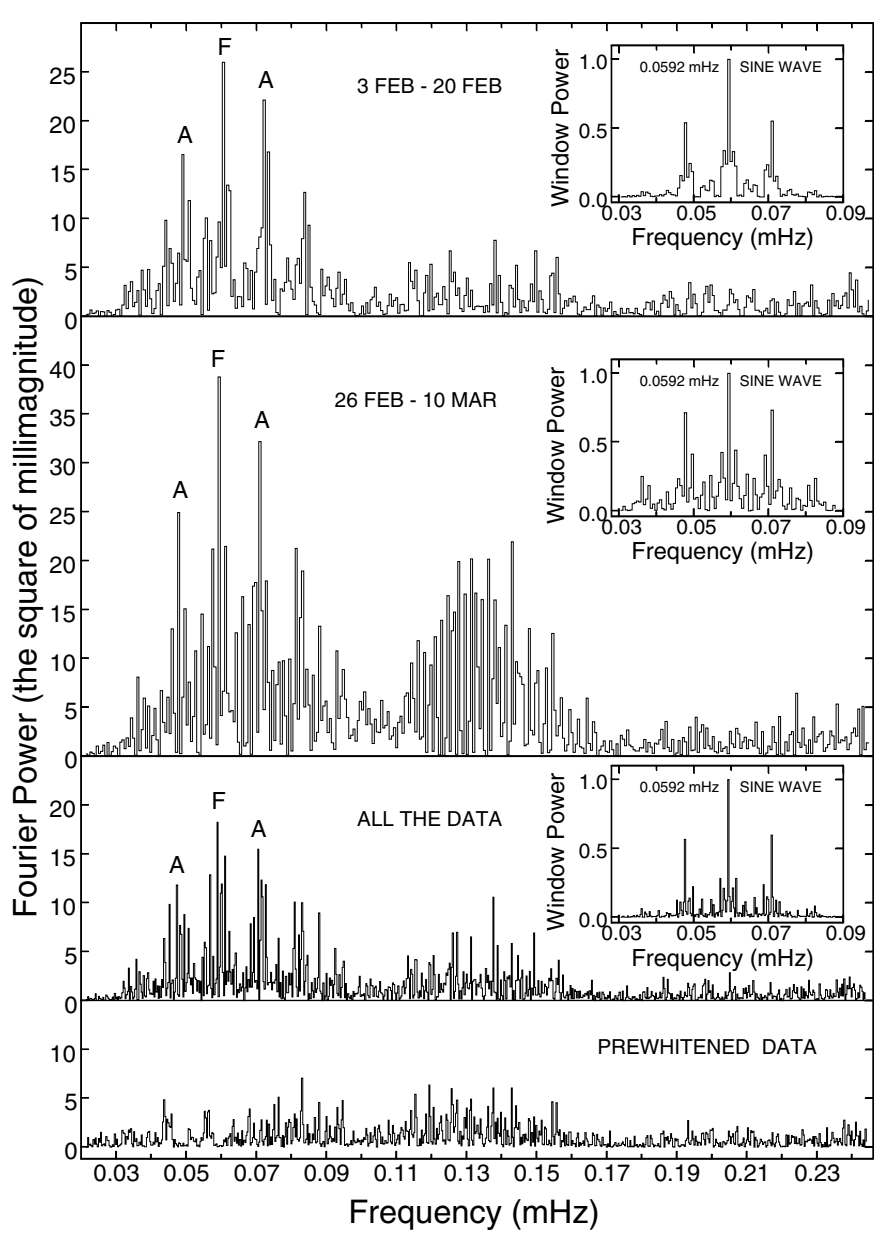

Fig. 5. Fourier power spectra of AT Cnc that are calculated using the data divided in two groups and using all the data together. The inserted frames represent the window functions calculated from sinusoidal signals sampled exactly as the real data. The two groups of the data reveal two nearly coherent oscillations at frequencies $0.0598 \pm 0.0003$ (3 February-20 February) and $0.0586 \pm 0.0003 \mathrm{mHz}$ (26 February-10 March). All the data sets together reveal that these oscillations have poor coherence during the whole observational time. The principal peaks are labeled with " $F$ " and one-day aliases are labeled with "A".

3 February and 20 February and $(0.0586 \pm 0.0003) \mathrm{mHz}$ within the time interval between 26 February and 10 March. We consider the error equal to half the frequency bin. Although the real frequency resolution for these data is somewhat worse (approximately by $10 \%$ and $30 \%$, accordingly) due to the small discrepancy between the time series length and the power of 2, nevertheless the frequency bins for the strictly periodic oscillation must coincide. The observed frequencies correspond to periods of $(4.65 \pm 0.03)$ and $(4.74 \pm 0.03) \mathrm{h}$, accordingly.

The large noise level visible between the principal peaks and the one-day aliases in the power spectra (Fig. 5) may denote the presence of one more oscillation with a similar frequency. To find this oscillation we attempted to exclude the main oscillation from the data. However, since the main oscillation is unstable, it seems impossible to fit the only sine wave to all the data. That is why we excluded two sine waves with frequencies of $0.0598 \mathrm{mHz}$ and $0.0856 \mathrm{mHz}$ and with appropriate amplitudes and phases from the first group and the second group of the data. Then we constructed a time series consisting of the prewhitened data. The power spectrum of these data is shown in the lower frame of Fig. 5. Obviously, the large amount of chaotically situated peaks at frequencies in the range $0.07-0.10 \mathrm{mHz}$ means that we were unable to exclude the main oscillation completely. However, at frequencies in the range $0.04-0.06 \mathrm{mHz}$ this power spectrum reveals two wide isolated structures which are one-day aliases of each other and therefore may indicate an additional oscillation.

The power spectra presented in Fig. 5 confirm the oscillation found in the average power spectrum (Fig. 4) at a frequency of $0.064 \mathrm{mHz}$. However, another oscillation at a frequency of $0.141 \mathrm{mHz}$ shows only the comb of peaks without signs of a structure resembling the window function and, therefore, may be aperiodic, i.e. the corresponding peak in the average power spectrum might be caused by flickering. To resolve this question, we applied another method for the search and for analysis of periodic oscillations in noisy data. The power spectrum can be obtained by means of a sine wave fit (SWF) to data folded and grouped into bins according to the phase of a trial period, using the method of least squares. We made several numerical experiments with artificial time series and made sure that such a method has a larger detection sensitivity of periodic signals and has a higher frequency resolution in comparison with usual Fourier power spectra calculated by means of an FFT algorithm. SWF power spectra seem also preferable in comparison with AoV (Analysis of Variance) periodograms which we used in the analysis of oscillations of the intermediate polar V709 Cas (Kozhevnikov 2001) and of the suspected cataclysmic variable NSV 2872 (Kozhevnikov 2003). The reason is that the test statistic $\Theta_{\mathrm{AoV}}$ is sensitive not only to an oscillation with the period coinciding with the trial period but also to oscillations with multiple periods if they are present in the folded lightcurve. This creates additional noise in the AoV periodogram.

The SWF power spectra are presented in Fig. 6. As in the case of Fourier power spectra (Fig. 5), these power spectra reveal distinct pictures resembling the window functions in the frequency range $0.03-0.09 \mathrm{mHz}$. The oscillation frequencies corresponding to the principal peaks in the upper and middle frames are $(0.0598 \pm 0.0002)$ and $(0.0586 \pm 0.0002) \mathrm{mHz}$. We consider the error equal to half the peak width between half intensity points. The corresponding periods are $(4.65 \pm 0.02)$ and $(4.74 \pm 0.02) \mathrm{h}$ and agree with the periods obtained from the Fourier power spectra. In addition, the SWF power spectra at frequencies in the range $0.11-0.14 \mathrm{mHz}$ in the upper and lower frames of Fig. 6 reveal the structures resembling the window functions though they are poorly distinguishable in the noise. In this case, we cannot differentiate which of the two distinguishable multicomponent structures represents the principal peak. In the spectrum calculated from the data obtained in the time interval between 3 February and 20 February the multicomponent structure at a frequency of $(0.1141 \pm 0.0010) \mathrm{mHz}$ seems somewhat more powerful whereas in the power spectrum calculated from all the data the multicomponent structure at a frequency of $(0.1256 \pm 0.0010) \mathrm{mHz}$ has a slightly larger height. We consider the error roughly equal to half the width 


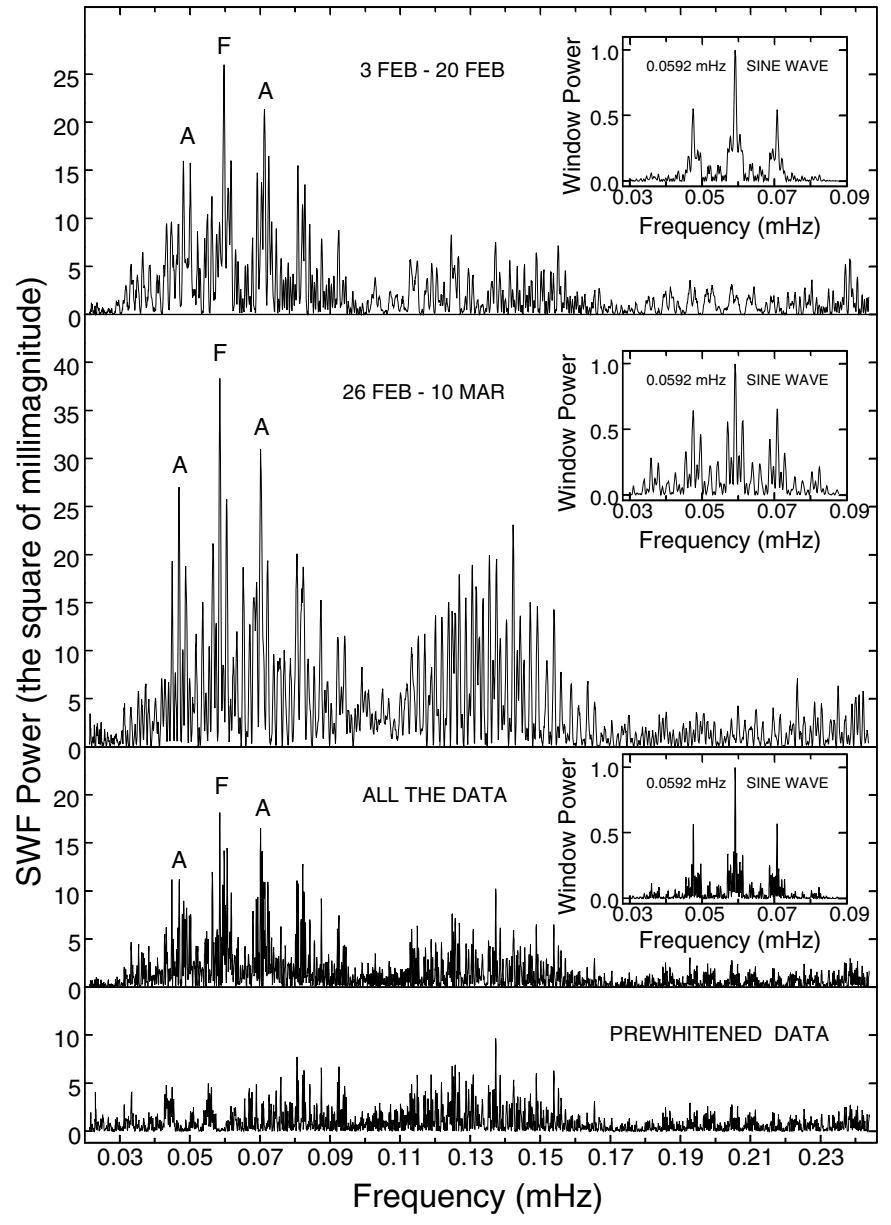

Fig. 6. SWF power spectra of AT Cnc that are calculated using a sine wave fit to folded lightcurves. Steps in change of trial frequencies are $0.00005 \mathrm{mHz}$. The forms of these power spectra in the frequency domain in the range $0.03-0.09 \mathrm{mHz}$ are similar to the forms of the Fourier power spectra (Fig. 5). In addition, the SWF power spectra in the upper and lower frames reveal a weakly coherent oscillation at a frequency of $(0.1141 \pm 0.0010)$ or $(0.1256 \pm 0.0010) \mathrm{mHz}$.

of the multicomponent structure. The presence of these structures confirms the existence of the second oscillation. As in the case of the frequencies in the range $0.03-0.09 \mathrm{mHz}$, the multicomponent structures mean that the corresponding oscillation is also quasiperiodic and of poor coherence. The period corresponding to these frequencies can be roughly evaluated equal to $2.3 \mathrm{~h}$. As in the case of the Fourier power spectrum, the SWF power spectrum of the prewhitened data (the lower frame of Fig. 6) at frequencies in the range $0.04-0.06 \mathrm{mHz}$ reveals two wide structures which may indicate another weakly coherent oscillation.

As the Fourier power spectra and SWF power spectra reveal (Figs. 5 and 6), the principal peaks and one-day aliases have multicomponent structures which demonstrate that the observed oscillations are unstable. We have performed several numerical experiments with artificial time series and made sure that such structures cannot be obtained when the oscillation period undergoes monotonous changes. Artificial power spectra similar to the observed power spectra can be obtained if the oscillation phases undergo random jumps from night to

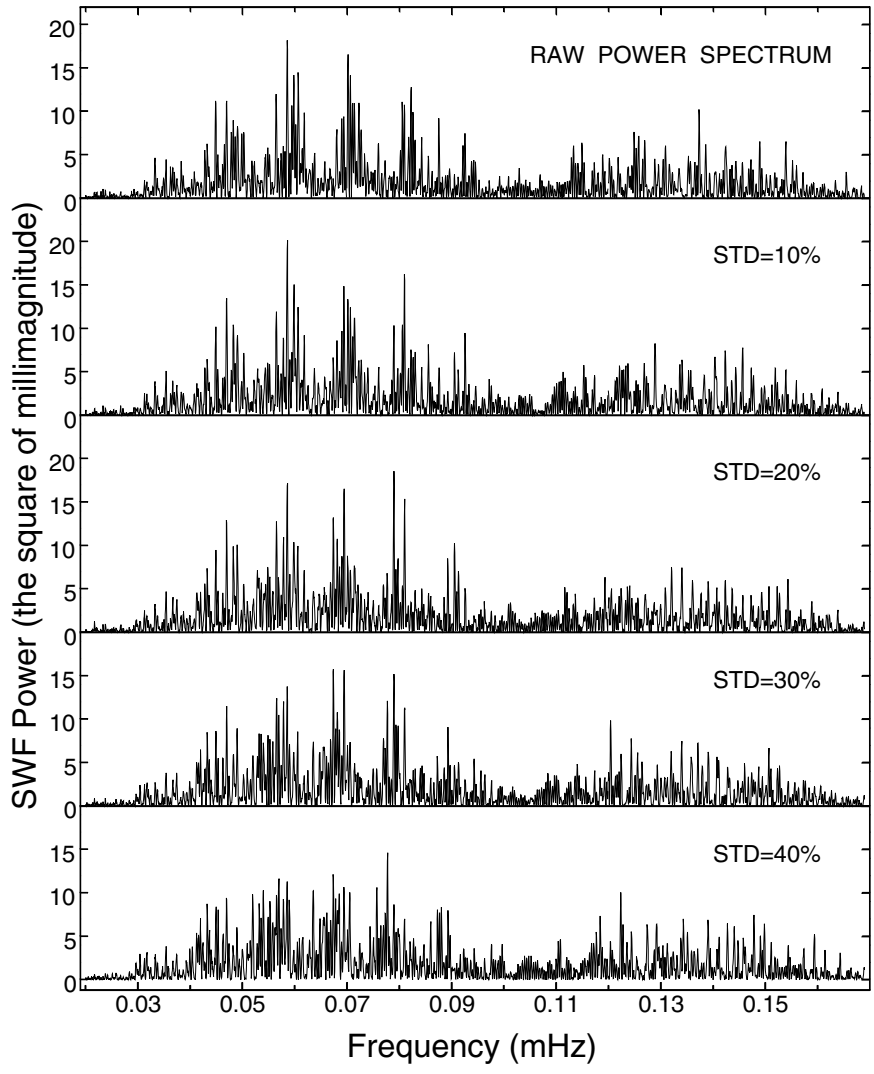

Fig. 7. SWF power spectra of AT Cnc that are obtained by changing the time of the beginning of the observations of each night randomly with a standard deviation of $10 \%, 20 \%, 30 \%$ and $40 \%$ of the observed period which equals $4.7 \mathrm{~h}$. The upper frame presents the SWF power spectrum without changes of the observational time. This numerical experiment shows that the observed oscillation is poorly coherent with a phase wobbling of about $10 \%-20 \%$ of the oscillation cycle.

night. In order to find out what phase wobblings might cause the observed appearance of the power spectra, we performed several numerical experiments with the real observational data. Figure 7 presents the SWF power spectra calculated from all the data by changing the times of the beginning of the observations of each night randomly. As seen in Fig. 7, the random jumps of the oscillation phases with a standard deviation of $10 \%$ of the observed period (which equals $4.7 \mathrm{~h}$ ) do not cause an appreciable modification of the power spectrum whereas the random jumps of the oscillation phases with a standard deviation of $40 \%$ considerably destroy its structure. This confirms the reality of the two oscillations and shows that the completely random oscillation phases (as in the case of aperiodic variations) cannot produce certain structures resembling the window functions in power spectra. Thus, we can consider that the observed oscillations have a poor phase coherence with a phase wobbling of about $10 \%-20 \%$ of the oscillation cycle.

It is obvious that artificial randomization of oscillation phases must erase any structures caused by periodic oscillations in power spectra. However, when we performed analogous numerical experiments with the prewhitened data, we found out that randomization of the oscillation phases cannot erase the two wide structures visible in the power spectra of the 
prewhitened data at frequencies in the range $0.04-0.06 \mathrm{mHz}$ (the lower frames of Figs. 5 and 6). When we changed the times of the beginning of the observations randomly, we found that these structures changed their width and height only slightly. In addition, they changed their location in frequency. This definitely testifies against periodic oscillation at frequencies corresponding to these structures in the power spectra of the prewhitened data and means that detection of this oscillation may be spurious.

Using all the observational data together, we cannot find the pulse shape of the oscillations since the individual oscillation cycles will be considerably displaced from each other in the folded lightcurve due to the unstable phase. The situation seems better when we consider two groups of the data separately. In these cases the power spectra presented in Figs. 5 and 6 reveal the clear principal peaks. The lightcurves folded with the corresponding periods are shown in Fig. 8. Both lightcurves reveal similar pulse shapes, which are asymmetric and characterized by wider minima in comparison with maxima. Obviously, these folded lightcurves present the same oscillation, although the degree of coherence of this oscillation seems weak over large time intervals.

\section{Discussion}

The oscillation with a period of $4.7 \mathrm{~h}$ discovered by our observations in AT Cnc shows approximately the same semiamplitude as the oscillation discovered by Nogami et al. (1999) and may be the same oscillation. However, its period significantly differs from the period candidates reported by Nogami et al. A reason for this discrepancy may be that the photometric observations obtained by Nogami et al. had insufficient length. The total duration of their photometric observations obtained during three nights is only $13.2 \mathrm{~h}$. When the oscillation phases randomly wander, this may lead to an error of the measured period. Especially it seems true when the length of the observation during each night is less than the oscillation period, and most of the photometric observations obtained by Nogami et al. were made just so. Since we found out that the oscillation with a period of $4.7 \mathrm{~h}$ is quasiperiodic, it cannot be caused by the rotating magnetic white dwarf. Hence, the assumption made by Nogami et al. that AT Cnc may be an intermediate polar seems groundless. The same reason, i.e. the quasiperiodicity of the oscillation, eliminates the possibility that this oscillation may represent the orbital period of the system. Therefore, it seems quite reasonable to consider that AT Cnc exhibits a kind of superhump. The asymmetric shape of the oscillation (Fig. 8) is also evidence supporting the superhump interpretation (see, e.g., Fig. 5 of Retter et al. 1997 and Fig. 4 of Patterson et al. 1997). If this interpretation is true, then this is the first detection of superhumps in a Z Camelopardalis-type dwarf nova.

Since different kinds of superhumps are detected in CVs depending on their brightness states, it is important to know the brightness state of AT Cnc during our observations. Unfortunately, at the time of the observations the magnitude of the comparison star was not measured with the aid of a standard star. Now, using the photo plate which was obtained earlier and two standard stars in the vicinity of AT Cnc (Misselt 1996),

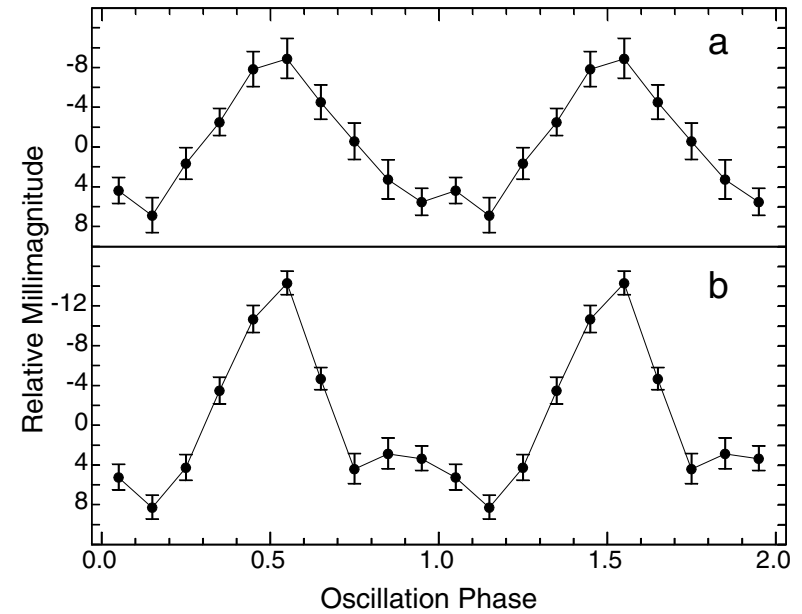

Fig. 8. Lightcurves of AT Cnc that are folded with a period of $4.65 \mathrm{~h}$ for the data obtained in the time interval between 3 February and 20 February (the frame a)) and with a period of $4.74 \mathrm{~h}$ for the data obtained in the time interval between 26 February and 10 March (the frame b)). Ten phase bins are used for each folded lightcurve.

we found the approximate $V$ magnitude of the comparison star equal to $12.9 \mathrm{mag}$. Then the approximate magnitude of AT Cnc was 13.6-13.7 mag during our observations. This magnitude corresponds to the standstill of AT Cnc. In addition, we examined the VSNET observations of AT Cnc (Fig. 1) and made sure that AT Cnc remained in this rather rare long standstill with magnitudes in the range 13.0-13.9 between December 2002 and June 2003. Thus, the superhumps in AT Cnc were observed during its long standstill.

The standstill of the Z Camelopardalis-type dwarf novae ought to be considered as a normal brightness state, which is similar to the state of a novalike variable but not of an outburst. Hence, the observed superhumps in AT Cnc must be permanent superhumps unlike the superhumps observed in the SU Ursae Maioris stars during superoutburst. Then we have two possibilities. The observed superhumps may be positive superhumps or negative superhumps. The periods of the brightness variations which were obtained in the two sets of our data of AT Cnc are 4.65 and $4.74 \mathrm{~h}$. They display a remarkable difference with the orbital period found by Nogami et al. (1999), which equals $0.2011 \mathrm{~d}(=4.83 \mathrm{~h})$. This difference is in the range $1.9-3.7 \%$. The average superhump period is less by $2.8 \%$ than the orbital period. Hence, the superhumps observed in AT Cnc are negative. Of course, there may be an aliasing problem, which may give rise to doubts about such a conclusion when photometric data are sparsely distributed in time. We believe that the aliasing problem is absent from our data, since the principal peaks in the power spectra (Figs. 5 and 6) have significantly larger height in comparison with the one-day aliases. Moreover, the data divided into two groups reveal similar results, although the distributions of the observations in time for these groups are quite different.

Several permanent superhump systems reveal both types of superhumps simultaneously (Patterson 1999). However, the positive superhumps which are caused by apsidal precession of the eccentric accretion discs are basically found in stars with 
small mass ratio, with $q=M_{\text {donor }} / M_{\text {compact }}<0.33$. This tends to confine this phenomenon to stars of short orbital period, since those binaries must have low-mass secondaries. In addition, the theory tells us that the disc must be relatively large, and then its eccentricity can be excited due to gravitational perturbation from the secondary. This agrees with the observational fact that positive superhumps occur only when the stars are relatively luminous (Patterson et al. 1993). Positive superhumps appear in high-mass-transfer discs (those of nova-like variables and of nova remnants) in some systems with orbital periods up to $3.5 \mathrm{~h}$ (Skillman et al. 1998). As an extreme case, it is possible for eccentricity to be excited in the disc of a high mass transfer system with $q=1 / 3$ if the mass transfer rate is reduced, as thought to occur in VY Sculptoris systems (Murray et al. 2000; Montgomery 2001). A rare exception is TV Col. This star shows positive superhumps with a period of $6.3 \mathrm{~h}$ and has an orbital period of $5.5 \mathrm{~h}$. However, TV Col may be an extreme system containing an undermassive secondary evolved at the end of hydrogen burning. In addition, TV Col is an intermediate polar, and the disc size in this system may be larger than usual due to the strong magnetic field of the white dwarf (Montgomery 2001; Retter et al. 2003). The orbital period of AT $\mathrm{Cnc}$ is $0.2011 \mathrm{~d}(=4.83 \mathrm{~h})$ and the masses of the binary components are $M_{\text {compact }}=(0.9 \pm 0.4) M_{\odot}$ and $M_{\text {donor }}=(0.47 \pm 0.05) M_{\odot}($ Nogami et al. 1999$)$. Thus, AT Cnc turns out to have a large mass ratio in the range $0.32-1.04$, and positive superhumps in this system seem unlikely.

Stars having negative superhumps are not numerous. Such CVs are more heterogeneous. They display no obvious traits in common and span a huge period range from AM CVn at $0.28 \mathrm{~h}$ (Skillman et al. 1999) to TV Col at $5.2 \mathrm{~h}$ (Augusteijn et al. 1994). The amplitudes of such superhumps range over a factor of 200 (0.003-0.60 mag) (Harvey et al. 1995). The average difference between the orbital period and superhump period observed in AT Cnc is $2.8 \%$. Such a difference is typical of negative superhumps. Also the multicomponent structures of the principal peaks and one-day aliases in the power spectra of AT Cnc (Figs. 5 and 6) strikingly resemble the structures in the power spectrum of V503 Cyg which is a well-known negative superhumper (see Fig. 12 of Harvey et al. 1995). This means that the wandering of the period and phase of superhumps in AT Cnc resembles the wandering of the period and phase of superhumps in V503 Cyg though the appearance of the power spectra testifies to somewhat more unstable superhumps in AT Cnc. Thus, we do not see any discrepancy in regarding the observed brightness variations of AT Cnc as negative superhumps.

Retter et al. (2002a) speculated that each permanent superhump system may have both kinds of superhumps. Although the positive superhumps in AT Cnc seem unlikely due to the large mass ratio, nevertheless we can estimate a hypothetical positive superhump from the relation between the positive superhump excess and the negative superhump deficit which was found by Patterson (1999) for systems exhibiting both types of superhumps simultaneously. Patterson proposed that period deficits in negative superhumps are about half the period excesses in positive superhumps: $\epsilon_{-} \approx-0.5 \epsilon_{+}$, where $\epsilon=\left(P_{\text {superhump }}-P_{\text {orbital }}\right) / P_{\text {orbital }}$. However, Retter et al. $(2002 \mathrm{~b})$

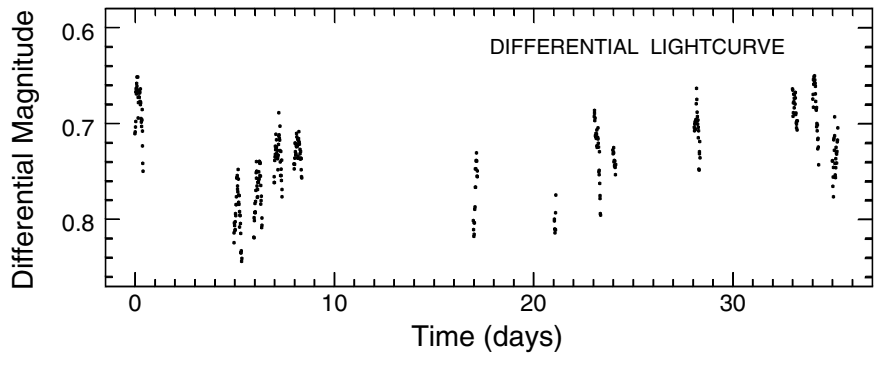

Fig. 9. Complete differential lightcurve of AT Cnc.

found a more precise relation in which the ratio $\phi=\epsilon_{-} / \epsilon_{+}$was a function of orbital period. From their Fig. 5 we can find that this ratio can be approximately in the range $0.32-0.44$ for systems having orbital periods of about 0.2 days. Then, given the observed negative superhump periods which are 4.65 and $4.74 \mathrm{~h}$ in the two sets of our data we have estimated the hypothetical positive superhump period of AT Cnc approximately in the range 5.0-5.4 h. One of the two wide structures visible at a frequency of $0.055 \mathrm{mHz}$ in the power spectra of the prewhitened data (lower frames of Figs. 5 and 6) is located within this range of periods. Another wide structure at a frequency of $0.044 \mathrm{mHz}$ might be the one-day alias of this possible signal. However, we are not convinced that these structures correspond to a real periodicity since artificial phase randomization does not erase them. As mentioned, Götz (1986) detected photometric variations of AT Cnc with a period of $0.2386913 \mathrm{~d}(=5.73 \mathrm{~h})$. This period seems too long, and it seems unlikely that it may represent the positive superhump period. In addition, we do not find any signs of this period in our data. Thus, our data do not allow us to draw a conclusion about the presence of positive superhumps in AT Cnc.

Unlike the positive superhumps explained by the prograde apsidal precession of the eccentric accretion disc, the negative superhumps can be explained by the retrograde precession of the line of nodes in an accretion disc tilted with respect to the orbital plane (Harvey et al. 1995; Skillman et al. 1998; Patterson et al. 2002). The superhump frequency is then the orbital sideband of the wobble frequency of the disc, $1 / P_{\text {prec }}$, i.e. $1 / P_{\mathrm{sh}}=1 / P_{\text {orb }}+1 / P_{\text {prec }}$. There may be photometric signs at the wobble frequency itself due to the periodic variation of the disc area. Unfortunately, in the case of AT Cnc the rather large instability of the superhump period allows us to establish only a wide range of the possible $P_{\text {prec }}$. This period may be in the range 5-12 d. Obviously, the wobble frequency of the disc cannot be detected in the de-trended data. That is why we constructed a time series including all our lightcurves without any de-trending. This time series is presented in Fig. 9. The Fourier power spectrum of the de-trended data is shown in Fig. 10. Although this spectrum reveals two prominent peaks with periods $11.9 \mathrm{~d}$ and $3.65 \mathrm{~d}$, which are close to the possible periods of the disc wobble, these peaks cannot be regarded as statistically significant. The highest peak with a period of $3.65 \mathrm{~d}$ is only two times as large as the surrounding noise level. Obviously, insufficient observational data does not allow us to find a period of brightness variations in the lightcurves of AT Cnc in this range of periods. 


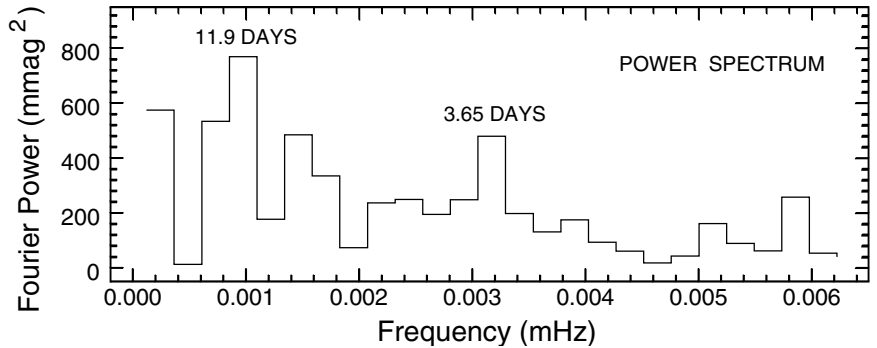

Fig. 10. Power spectrum of the complete differential lightcurve.

However, the wobble frequency of the disc may become perceptible in another orbital sideband, namely $2 / P_{\text {orb }}+1 / P_{\text {prec }}$, as, for example, is seen in DW UMa (Patterson et al. 2002). Given the superhump periods and the orbital period found by Nogami et al. (1999), we can estimate the frequency of this orbital sideband in the range $0.116-0.118 \mathrm{mHz}$. However, as seen in Fig. 8, the shapes of the superhumps in the folded lightcurves are largely nonsinusoidal and can show the second harmonic which can be in the frequency range $0.117-0.120 \mathrm{mHz}$. Obviously, due to the instability of the superhump period, the second orbital sideband of the wobble frequency of the disc proves to be practically indistinguishable from the second harmonic of the superhump variations. Moreover, as numerical experiments with artificial time series showed, it seems impossible to point out the locations of the corresponding peaks in the power spectra with tolerable accuracy due to the large instability of the oscillation phases. Then, the second peak at a frequency of $(0.141 \pm 0.026) \mathrm{mHz}$ (Fig. 4) and the comb of peaks in the range $0.11-0.16 \mathrm{mHz}$ (Figs. 5 and 6) may be caused by any of these two variations or by their simultaneous presence in the lightcurves.

The eccentricity of discs in the systems revealing positive superhumps is caused by gravitational perturbation of the secondary when the disc is sufficiently large. However, the origin of the disc tilt in the systems which show negative superhumps remains an open question (Wood et al. 2000). One possible reason is influence of the magnetic field of the primary (Murray 1998). White dwarf magnetism is demonstrably present in two negative superhumpers (TV Col and V709 Cas), and is arguable on the basis of kilosecond quasiperiodic oscillations (QPOs) in most of the others (Patterson et al. 2002). Magnetic white dwarf spin equilibrium should have $P_{\text {spin }} / P_{\text {orb }} \sim 0.1$ (King \& Lasota 1991; Wickramasinghe et al. 1991). Since the QPO periods in many CVs are within $20 \%$ of $P_{\text {orb }}$, it is possible that the QPOs reflect the underlying rotation of a magnetic white dwarf (Patterson et al. 2002). Although QPOs in the lightcurves of AT Cnc seem inconspicuous, some amplitude spectra (Fig. 3) reveal prominent peaks at higher frequencies which may indicate QPOs. Moreover, the average power spectrum (Fig. 4) reveals a broad hump at frequencies in the range 0.4-0.7 mHz. This hump is evidence of QPOs. The hump crest is located at a frequency of $0.55-0.60 \mathrm{mHz}$. This frequency corresponds to a period of $1700-1800 \mathrm{~s}$, which is remarkably close to $10 \%$ of the orbital period found by Nogami et al. (1999). Thus, like the negative superhumps in other CVs, the negative superhumps found in AT Cnc are accompanied by QPOs with periods which are close to $10 \%$ of the orbital period. This may indicate that this system contains a magnetic white dwarf though a strictly periodic oscillation typical of intermediate polars is not found in our observations.

However, the disc tilt caused by the magnetic field of the primary can co-rotate with the primary and cannot possibly generate the periods characteristic of negative superhumps since the primary star in a cataclysmic binary typically rotates several times faster than the binary. Another reason that might cause the disc tilt is the magnetic field of the secondary (Murray et al. 2002). Murray et al. suggested that, in such a case, negative superhumps could not be explained simply via the retrograde precession of a tilted disc, but in terms of changes in the disc vertical structure.

\section{Conclusions}

1. We have found brightness variations with an average period of $4.7 \mathrm{~h}$ in the optical lightcurve obtained during $97 \mathrm{~h}$ of observations of AT Cnc in February and March 2003.

2. The semiamplitude of these variations was observed approximately in the range 5-9 mmag, showing changes from night to night.

3. This signal varies in period and in phase on a time-scale of weeks, as is typical of superhumps.

4. The comparison with the orbital period obtained by Nogami et al. (1999) from the radial velocity measurements of AT Cnc shows that this signal can be indicative of negative superhumps since its period is approximately $3 \%$ shorter than the orbital period.

5. We have also found signs of a more unstable signal with a period of about $2.3 \mathrm{~h}$, which may represent the second harmonic of the 4.7-h signal or may represent the second orbital sideband of the wobble frequency of the tilted precessing accretion disc, i.e. $2 / P_{\text {orb }}+1 / P_{\text {prec }}$.

6. The average power spectrum of AT Cnc reveals a broad hump at frequencies in the range $0.4-0.7 \mathrm{mHz}$, which is evidence of QPOs. The hump crest corresponds to a period of $1700-1800 \mathrm{~s}$, which is remarkably close to $10 \%$ of the orbital period. This may indicate that this system contains a magnetic white dwarf creating the tilted accretion disc.

\section{References}

Augusteijn, T., Heemskerk, M. H. M., Zwarthoed, G. A. A., \& van Paradijs, J. 1994, A\&AS, 107, 219

Bendat, J. S., \& Piersol, A. G. 1986, Random Data Analysis and Measurement Procedures (John Wiley \& Sons, Ins.)

Götz, W. 1983, IBVS, 2363

Götz, W. 1985, IBVS, 2734

Götz, W. 1986, IBVS, 2918

Götz, W. 1988a, IBVS, 3066

Götz, W. 1988b, IBVS, 3208

Götz, W. 1990, Mitt. Veranderl. Sterne, 12, 60

Götz, W. 1991, Mitt. Veranderl. Sterne, 12, 111

Harvey, D., Skillman, D. R., Patterson, J., \& Ringwald, F. A. 1995, PASP, 107, 551

King, A.R., \& Lasota, J.-P. 1991, ApJ, 378, 674

Kozhevnikov, V. P. 2001, A\&A, 366, 891 
Kozhevnikov, V. P. 2003, A\&A, 398, 267

Kozhevnikov, V. P., \& Zakharova, P. E. 2000, in Proc. Euroconference on Disks, Planetesimals and Planets, ed. F. Garzon, C. Eiroa, D. de Winter, \& T. J. Mahoney, ASP Conf. Ser., 219, 381

Misselt, K. A. 1996, PASP, 108, 146

Montgomery, M. M. 2001, MNRAS, 325, 761

Murray, J. R., \& Armitage, P. J. 1998, MNRAS, 300, 561

Murray, J. R., Warner, B., \& Wickramasinghe, D. T. 2000, MNRAS, 315,707

Murray, J. R., Chakrabarty, D., Graham, A., Kramer, W., \& Kramer, L. 2002, MNRAS, 335, 247

Nogami, D., Masuda, S., Kato, T., \& Hirata, R. 1999, PASJ, 51, 115

Osaki, J. 1996, PASP, 108, 39

Patterson, J. 1994, PASP, 106, 209

Patterson, J. 1999, in Disk Instabilities in Close Binary Systems, 25 Years of the Disk-Instability Model, Proceedings of the Disk-Instability Workshop held on 27-30 October, 1998, ed. S. Mineshige, \& J. C. Wheeler, Front. Sci. Ser., 26 (Universal Academy Press, Inc.), 61

Patterson, J., Thomas, G., Skillman, D. R., \& Diaz, M. 1993, ApJS, 86,235
Patterson, J., Kemp, J., Saad, J., et al. 1997, PASP, 109, 468

Patterson, J., Fenton, W. H., Thorstensen, J. R., et al. 2002, PASP, 114, 1364

Retter, A., \& Naylor, T. 2000, MNRAS, 319, 510

Retter, A., Leibowitz, E. M., \& Ofek, E. O. 1997, MNRAS, 286, 745

Retter, A., Chou, Y., \& Bedding, T. 2002a, in The Physics of Cataclysmic Variables and Related Objects, ed. B. T. Gansicke, K. Beuermann, \& K. Reinsch, ASP Conf. Ser., 261, 527

Retter, A., Chou, Y., Bedding, T. R., \& Naylor, T. 2002b, MNRAS, 330, L37

Retter, A., Hellier, C., Augusteijn, T., et al. 2003, MNRAS, 340, 679

Skillman, D. R., Harvey, D. A., Patterson, J., et al. 1998, ApJ, 503, L67

Skillman, D. R., Patterson, J., Kemp, J., et al. 1999, PASP, 111, 1281

Wickramasinghe, D. T., Wu, K., \& Ferrario, L. 1991, MNRAS, 249, 460

Wood, M. A., Montgomery, M. M., \& Simpson, J. C. 2000, ApJ, 535, L39

Young, A. T., Genet, R. M., Boyd, L. J., et al. 1991, PASP, 103, 221 